\title{
Effect of Trunk Extensor Muscle Fatigue on Postural Stability in Healthy Young Adults
}

\author{
Isha Akulwar' ${ }^{1}$, Saili Mulgaonkar ${ }^{2}$
}

\begin{abstract}
Objective: To determine the effect of trunk extensor muscle fatigue on postural stability in healthy young adults using clinical measures. Design: Prospective, experimental, within subject, repeated measures design. Setting: Tertiary care hospital and research centre. Participants: 70 healthy adults in the age group of 20 to 25 years, volunteer sample. Intervention: Fatigue of trunk extensor muscles was induced by repeated dynamic contractions performed to maximum exhaustion. Postural stability was assessed under three different conditions viz. No fatigue, Fatigue and Recovery. Main Outcome Measures: One-leg standing balance test and Functional reach test were used to assess static and dynamic postural stability respectively. Results: Wilcoxon matched-paired signed ranked test was used for comparison of postural stability in No fatigue and Fatigue condition with p-value $<.001$. One-leg standing balance test showed a significant decrease from No fatigue $(15.16+8.47$ seconds $)$ to Fatigue $(5.89+5.7$ seconds $)$ condition. Functional reach test values showed a significant decrease from No fatigue condition $(12.34+2.61$ inches) to Fatigue condition $(12.27+2.6$ inches). Mean recovery time was 12.5+ 3.27 minutes for One-leg standing balance test and $11.93+3.22$ minutes for the Functional reach test. Further analysis using Mann Whitney test showed no significant difference $(P=.288)$ between the recovery time of one-leg standing balance test and functional reach test. Conclusions: Trunk extensor fatigue significantly affects static and dynamic postural stability in healthy young adults. Complete recovery takes $12.5+3.27$ minutes for static and $11.93+3.22$ minutes for dynamic postural stability. Results of this study stress the importance of intact trunk extensor muscle function on the regulation of static and dynamic postural stability in healthy young adults. However, more studies are required to determine the effect of trunk extensor muscle fatigue using other clinical measures and in individuals with neuromuscular disorders.
\end{abstract}

Keywords: Postural stability, Trunk extensor, Fatigue

\section{Introduction}

Postural control is a complex task that requires the integration of visual,vestibular and somatosensory inputs from all over the body to assess the position and motion of the body in space and the ability to generate forces to control body position. ${ }^{1}$ Thetask of maintaining upright balance is a critical component in performing most daily activities.Numerous studies $2,3,4,5,6,7,8,9,10,11,12,13,14,15$ have investigated the effects of muscular fatigue on the regulation of bipedal quiet upright standing.Generally, a deterioration of postural control with muscular fatigue was reported.Considering postural control as a sensorimotorprocess $^{16}$ (e.g., Schmidt, 1975), it is conceivable thatmuscular fatigue, known to alter the peripheral proprioceptivesystem, the central processing of proprioception but also the force-generating capacity ${ }^{17}$ (e.g., Taylor et al.,2000), affects both the sensory and the motor side of the process. There is substantial evidence on the detrimental effect of fatigue on postural control in older adults. ${ }^{6}$ However previous research on healthy young adults has been inconsistent as some studies have shown that postural control in quiet standing and during various dynamic perturbations can be maintained during muscle fatigue. ${ }^{18,19}$ Muscle fatigue has been proposed to cause a change in postural control strategy and several researchers have posited that changes in postural control do not necessarily imply a decrease in postural stability. ${ }^{17}$ Most of the investigations cited above referred to postural control following lower limb efforts or general body fatigue. In recent years, a growing number of studies have reported increased postural sway during quiet standing with muscle fatigue localized at the lower back. ${ }^{7,8,910,1112,13,14,15}$ All of these studies assessed postural stability using laboratory measures such as a force platform, however evidence using clinical measures of postural stability is lacking. Hence the purpose of this study was to determine the effect of trunk extensor muscle fatigue on static and dynamic postural stability using clinical measures in healthy young adults.Based on the findings from previous research, we hypothesized that localized fatigue of trunk extensor muscles would significantly reduce static and dynamic postural stability in healthy young adults. Although, the vast majority of postural stability research has been on doubleleg stance, periods of single-leg stance occur frequently within many activities of daily living. Also, postural instability increases during single-leg stance, thus, it can be speculated that the increased challenge of maintaining single-leg equilibrium may better elicit postural stability differences existing after fatigue. Functional reach test measures dynamic balance during a functional task of reaching while maintaining trunk stability. Considering the importance of balance in activities of daily living, functional balance tests such as One-leg standing balance test and Functional reach test were used as appropriate outcome measures for this study.

The effects of muscle fatigue appear to elicit a transient means of postural instability however the recovery of postural stability after fatiguing exercise has not been extensively studied.Thus another purpose of the study was to determine the time period for which the effect of trunk extensor muscle fatigue on postural stability would persist.

\section{Methods}

This was a prospective, experimental study with withinsubject design. 70 healthy adults in the age group of 20-25 years volunteered to participate in this study. All the subjects were physically active university students.Individuals with back or lower limb pain, any musculoskeletal or neurological condition or vestibular impairment were excluded from participation. All the subjects provided their informed consent. The procedures followed protocol and 


\section{International Journal of Science and Research (IJSR) \\ ISSN (Online): 2319-7064}

Index Copernicus Value (2015): 78.96 | Impact Factor (2015): 6.391

accord with the ethical standards of the institutional review board.Postural stability was assessed under three conditions:

Condition 1: No fatigue

Condition 2: Fatigue(immediately after fatigue has set in)

Condition 3: Recovery (after every five minutes till complete recovery to No fatigue condition)

The No fatigue condition served as a control condition.Oneleg standing balance test (OLST) ${ }^{20}$ and Functional reach test $(\text { FRT })^{21}$ was used to assess static and dynamic postural stability respectively. Order of testing was OLST followed by FRT under all three experimental conditions. The dominant leg was first determined by asking the subject to kick a ball placed on the floor in front of him/her and the 'kicking limb' was considered as the 'dominant leg'. For OLST, subject stood barefoot on a firm stable surface on the dominant leg with the other leg raised, arms crossed over the chest. Test was performed under eyes closed condition to avoid vision from interfering with the task of postural control. Time of one leg standing was recorded in seconds using a stopwatch. Test was terminated when the subjectused his arms (i.e., uncrossed arms), used the raised foot (moved it toward or away from the standing limb or touched the floor), moved the weight-bearing foot to maintain balance (i.e., rotated foot on the ground), or opened eyes. Three trials were given and best of the three trials was taken as the final reading. (Bohannon R, Larkin P, 1984).FRT was performed by the subject standing next to but not touching a wall with dominant arm closer to the wall at 90 degrees of shoulder flexion with a closed fist. Subject selected a comfortable stance with feet shoulder width apart. To keep foot positions and base of support constant during re-assessments in all three conditions footprints were marked .The subject was asked to reach forward as far as possible without taking a step. The distance between the start and the end position was measured using the head of the third metacarpal as the reference point and was recorded in inches. Three trials were given and the average of the three trials was taken as the final reading (Pamela Duncan; 1990).To induce fatigue of trunk extensor muscles subject was made to perform dynamic trunk extensions until maximum exhaustion (Vuillerme et al.,2007).For this task, subject lay prone on a bench with the upper body unsupported in the horizontal plane and the lower limbs secured to the bench with straps at the hips, knees and ankles. During the test the armswere held across the chest. The subject was instructed to raise his/her upper body toa horizontal position with the head and neck in neutral position and then lowering it back down. A digital metronome set at 40 beeps/min was used to ensure all the subjects performed the extensions at a consistent rate. Subject was instructed to perform the extension movement as many times as possible till he/she was unable to continue due to muscle fatigue. Verbal encouragement was givento ensure that the subject worked maximally. After termination of this task postural stability was assessed immediately (Fatigue condition) using OLST and FRT as described earlier. The subjective exertion level for the fatiguing task was assessed through the Borg 6-20 $\operatorname{scale}^{21}$ (Borg 1990). For recovery of induced trunk extensor muscle fatigue, rest was given in a supported position. To observe recovery in postural stability (Recovery condition)OLST and FRTwere performed every 5 minutes till the complete recovery to $\mathrm{No}$ fatigue condition was achieved. Subjects were asked to refrain from any strenuous physical activity for past 48 hours. Considering the time-of-day effects on fatigue and postural control, assessment was carried out during the same time of the day (afternoon) for all the subjects. All the tests were administered by the same investigator and in an identical manner under all three conditions. Data thus collected was subjected to statistical analysis.

\section{Results}

Subjects' mean age, height and BMI were $21.77 \pm 1.55$ years, $157.94 \mathrm{~cm}$ and $21.75 \mathrm{~kg} / \mathrm{m}^{2}$ respectively. Mean rating of perceived exertion for the fatiguing task was $17.72 \pm 1.59$ on Borg scale 6-20.Variables distribution was tested using Kolmogorov-Smirnov test which exhibited significant departures from normality, and therefore nonparametric tests were used for all the analyses. Statistical significance was set at $\mathrm{P}<0.001$. Wilcoxon matched- paired signed rank test was used to compare postural stability in No fatigue and Fatigue condition.OLST showed a decrease from $\mathrm{No}$ fatiguecondition (15.16 \pm 8.47seconds, (95\% confidence interval (CI), 13.13-17.18) to Fatiguecondition (5.89 5.7 seconds)(95\% CI, 4.53-7.25), (one-tailed $\quad P<.0001)$. FRTshowed a decrease fromNo fatiguecondition (12.34 + 2.61 inches) (95\% CI, 11.71-12.96) toFatiguecondition (12.27 \pm 2.6inches)(95\% CI, 11.65-12.89),(one-tailed $P<$ $0.0001)$. The results indicate that postural stability was significantly reduced in the Fatigue condition as compared to the No Fatigue condition.

To determine the recovery period for postural stability, mean values ofthe consecutive readings for OLSTand FRTtaken during the Recovery condition were compared with the $\mathrm{No}$ fatigue condition. It was observed that mean recovery time for OLSTwas $12.5+3.27$ minutes and $11.93 \pm 3.22$ minutes for the FRT. Further analysis using Mann Whitney test showed no significant difference between the recovery time of one-leg standing test and FRT (two-tailed $P=0.288$ ).

\section{Discussion}

The purpose of the present study was to determine the effect of trunk extensor muscle fatigue on postural stability in healthy young adults using clinical measures. Trunk extensor muscle fatigue significantly affected static and dynamic postural stability as indicated by reduction inOLST and FRTrespectively from No fatigueto Fatiguecondition.This result confirms our hypothesis in accordance with previous reports.Trunk extensors are physiologically postural muscles being rich in type I muscle fibres. $^{23,24}$ The erectorsspinae and multifidi are found to be tonicallyactive in quiet standing and the erectoresspinae contract eccentrically to control trunk flexion from a standing position and also when the head or upper limb is moved forward. Therefore fatigue in these muscles may lead to difficulties in maintaining upright posture. Neuromuscular fatigue represents a major constraint on the postural control system.It has been suggested from studies that muscular strength and sensory detection are two important factors in fatigue and postural control. ${ }^{11,12,13,25,26}$ Trunk muscle fatigue was shown to impair the ability to sense a change in lumbar

\section{Volume 6 Issue 7, July 2017 www.ijsr.net}




\section{International Journal of Science and Research (IJSR) \\ ISSN (Online): 2319-7064}

Index Copernicus Value (2015): 78.96 | Impact Factor (2015): 6.391

position, to delay the reaction time of the muscles in response to a sudden load, to reduce the force generating capacity and to increase its variability.Single-leg stance is associated with decreased inherent stability, most likely as a result of the required reorganization of the center of gravity (COG) over a short and narrow base of support. The small margin of sway permitted to remain in equilibrium (vertical projection of COG over base of support) requires accurate and prompt sensory information. Furthermore, single-leg stance reduces the quantity of useful and accurate somatosensory information (both proprioceptive and plantar cutaneous) available to the postural control system. The decrease in somatosensory information, coupled with decreased inherent stability, may result in a higher reliance on visual inputs and proprioceptive inputs from other postural muscles of the body during one leg stance. In this study, OLST was performed under eyes closed condition, thus, vision was removed from contributing to the task of postural control. The vestibular system was not likely affected by neuromuscular fatigue as the subjects stood on a firm surface and head was aligned upright. Additionally, studies by Allum et al (1998) $)^{27}$ and Bloem et al $(2002)^{28}$ have indicated that trunk and hip proprioceptive inputs may provide the triggers for most balance corrections. However, proprioceptive feedback of trunk position is impaired by lumbar extensor fatigue as reported by Taimela et al(1999) ${ }^{25}$, Davidson et al(2004) ${ }^{4}$. Researchers have studied the different postural control strategies and kinematic patterns activated during one leg stance. ${ }^{29}$ In all these investigations, the ankle dominated as the source of corrective action whereas the trunk appeared to be the least important source of corrective action. It can be speculated that the higher inertia associated with the trunk may preclude it from contributing to the quick adjustments necessary for singleleg stance equilibrium.Considering the lesser role of trunk musculature strength and more reliance on proprioceptive inputs from these muscles during one leg stance under eyes closed condition, the decrease in static stability during OLST as observed in this study can therefore be explained by the diminished proprioceptive feedback from fatigued trunk extensors. This explanation can further be reinforced by studies which suggest that altered neck proprioception due to neck extensor fatigue affects postural control during quiet stance. ${ }^{30,31,32}$ In this study, FRT was used as a measure of dynamic stability. Voluntary arm movements in standing induce dynamic postural perturbation and to minimize these planned postural perturbations, the central nervous system uses anticipatory activation of the trunk and leg muscles.(Aruin\&Latash, 1995a). Recently few investigators ${ }^{33,34,35,36,37,38}$ have studied the fatigue-induced adaptive changes of anticipatory postural adjustments (APAs) during unilateral or bilateral upper limb movements. Muscle fatigue has been shown to result in decrease in the level of muscle excitation and early onset of APAs.Early APA onset may enhance postural stability by permitting a longer duration APA which can counteract fatigue-induced decreases in the force-producing capability of muscles that contribute to postural stability. It has been posited that fatigue-induced early onset of APA is a centrally mediated adaptation and may reduce the impact that fatigue has on the body during various dynamic perturbations. It is worth mentioning that all the aforementioned studies are largely based on the muscle activation patterns using electromyography whereas measures of postural stability have not beenadequately investigated. Among these, Vuillrme et al (2002) ${ }^{34}$ reported that lower limb muscle fatigue induced decrease in postural stability before movement execution, however postural stability was not assessed during movement execution. Strang\& Berg (2007) foundno effect on postural stability during the focal movement after fatigue induced by dead lift exercise. ${ }^{37}$ In contrast with these results we found a significant reduction in postural stability during forward reach task after trunk extensor fatigue.Indeed, it is well documented that fatigue in the extensor muscles of trunk reduces trunk's local dynamic stability. ${ }^{39,40}$ Reduction in paraspinal muscle stiffness and force generating capacity, modified trunk muscle recruitment with increased coactivation of the trunk flexor musculature, delay in feedback responses, modified paraspinal muscle reflex response with fatigue have been suggested as potentially influencing neuromuscular control of movement and spinal stability. In line with these findings, reduction in postural stability during forward reach test after trunk extensor fatigue as observed in this study can be attributed to a reduced control over the trunk. To our knowledge, the current study is the first one to investigate the effect of trunk extensor fatigue on dynamic postural stability during voluntary movement. Considering the limited literature available in this regard, more studies are warranted to explore these results further.Muscle fatigue can be defined as "an exercise-induced reduction in the ability of muscle to produce force or power whether or not the task can be sustained". $50 \%$ or more decreased maximum voluntary contraction resulted in balance deterioration in previous studies. The fatigue protocol used in this study was designed to induce maximum fatigue of trunk extensor muscles.Subjects were asked to perform the task till they were unable to continue due to muscle fatigue and also, verbal encouragement was given to ensure that they worked maximally. We considered a rating of 17 (very hard) and above on the Borg RPE scale 6-20 as adequate fatigue which reportedly corresponds to approximately more than $90 \%$ $\mathrm{VO}_{2}$ max and maximum heart rate. ${ }^{22}$ The fatigue protocol produced desired fatigue in all the subjects as indicated by the reported rating of $17.72 \pm 1.59$ on RPE scale. The mean recovery time for fatigue-related changes in postural stabilitywas12.5 \pm 3.27 minutes for OLST and 11.93 \pm 3.22 minutes for the FRT with no significant difference between the two tests. Few researchers have investigated the recovery rates after trunk extensor muscle fatigue in healthy young adults.It was found that postural sway parameters did not return to pre-fatigue values after 30 minutes (Davidson et $\mathrm{al}^{5}$, Pline et $\mathrm{al}^{10}$ ) for quiet standing and 20 minutes for one leg balance test (Parreira et $\mathrm{al}^{9}$ ). In yet other studies ${ }^{42.43}$ using different muscle groups, Yaggie JA, McGregor SJ, 2002 reported 20 minutes of recovery for unilateral stance and lean test after lower extremity fatigue and Nardone et al,1997 reported that fatigue effects during quietstanding lasted until about 15 minutes from the end of fatiguing treadmill exercise.The disparities observed in recovery rates across these studies can be likely due to different types of fatigue protocols and outcome measures used. Again it should be noted that all the above cited studies are based on force platform measures and the relatively short period of recovery observed in the present study can be due to the difference in the sensitivity of force platform and clinical 


\section{International Journal of Science and Research (IJSR) \\ ISSN (Online): 2319-7064}

Index Copernicus Value (2015): 78.96 | Impact Factor (2015): 6.391

measures in detecting subtle differences in postural stability.Along with the existing evidence results of this study stress the importance of intact trunk extensor muscle function on the regulation of postural stability and thus can have important implications in ergonomic, clinical and rehabilitative areas.For results to be applicable in these areas we recommend future research.Nonetheless findings of this study suggest that trunk extensor fatigue could potentially lead to loss of balance which can be a major concern for certain occupations, neuromuscular conditions and geriatric population.Also, these results can contribute in designing balance intervention program. An important aspect of this study is that we used more functional outcome measures which are clinically applicable outside laboratory settings. Considering the significant effect of fatigue on postural stability which lasts for $\sim 12$ minutes, clinical assessment of postural stability should be performed before or after 12-15 minutes of assessment or exercises of trunk extensors to eliminate the effect of fatigue on assessment of postural stability.

\section{Study Limitations}

One of the possible limitations of the study is the subjective nature of the criteria used for muscle fatigue. Another limitation is that reactive postural control in response to external perturbations was not assessed in this study. We recommend future studies focusing on more objective tools and methods and incorporating additional measures of postural control.

\section{Conclusions}

Trunk extensor fatigue significantly reduces static and dynamic postural stability in young healthy adults as observed from one leg standing balance test and functional reach test.Effect of fatigue takes $12.5 \pm 3.27$ minutesfor static and $11.93 \pm 3.22$ minutes fordynamic postural stability to recover completely. We recommend more studies using other clinical measures of postural stability and in individuals with neuromuscular disorders.

\section{References}

[1] Massion, J. Movement, posture and equilibrium: interaction and coordination. Prog. Neurobiol. 1992, 38, 35-56.

[2] Adlerton A., Moritz U. Does calf-muscle fatigue affect standing balance? Scand J Med Sci Sports. 1996 Aug;6(4):211-5.

[3] Adlerton AK, Moritz U, Moe-Nilssen R. Forceplate and accelerometer measures for evaluating the effect of muscle fatigue on postural control during one-legged stance. Physiother Res Int. 2003;8(4):187-99

[4] Davidson B., Madigan M., Nussbaum M. Effects of lumbar extensor fatigue and fatigue rate on postural sway. European Journal of Applied Physiology.2004 Oct;93(1-2):183-9.

[5] Davidson B., Madigan M., Nussbaum M., Wojcik L. Effects of localized muscle fatigue on recovery from a postural perturbation without stepping. Gait Posture. 2009;29:552-7.
[6] Helbostad J., Sturnieks D., Menant J., Delbaere K. Lord S., Pijnappels M. Consequences of lower extremity and trunk muscle fatigue on balance and functional tasks in older people: A systematic literature review. BMC Geriatr. 2010;10: 56.

[7] Kahlaee A., Bahrpeyma F., Esteki A. The effect of fatigue and instability on postural control parameters in standing posture in healthy adults and patients with chronic low back pain. Zahedan Journal of Research in Medical Sciences, 2012 Aug;14(6): 17-22

[8] Lin D, Nussbaum M, Seol H, Singh N, Madigan M, Wojcik L. Acute effects of localized muscle fatigue on postural control and patterns of recovery during upright stance: influence of fatigue location and age. Eur J Appl Physiol. 2009;106:425-34.

[9] Parreira R., Amorim C, Gil A., Teixeira D., Martin Bilodeau M., Silva R. Effect of trunk extensor fatigue on the postural balance of elderly and young adults during unipodal task. Eur J Appl Physiol. 2013 March

[10] Pline K., Madigan M., Nussbaum M. Influence of fatigue time and level on increases in postural sway. Ergonomics.15 December 2006; 49(15):1639-164.

[11] Vuillerme N, Anziani B, Rougier P. Trunk extensor muscles fatigue affects undisturbed postural control in young healthy adults. Clinical Biomechanics.2007;22(5):489-94.

[12] Vuillerme N., Pinsault N. Re-weighting of somatosensory inputs from the foot and the ankle for controlling posture during quiet standing following trunk extensor muscles fatigue. Exp Brain Res.2007;183:323-327

[13] Vuillerme N., Pinsault N., Chenu O., Fleury A., Payan Y., Demongeot J., Postural destabilization induced by trunk extensor muscles fatigue is suppressed by use of a plantar pressure-based electro-tactile biofeedback. European Journal of Applied Physiology 2008

[14] Vuillerme N., Pinsault N. Vestibular and neck somatosensory weighting changes with trunk extensor muscle fatigue during quiet standing. Exp Brain Res (research note, published online 25 December 2009).

[15] Wilson E., Madigan M., Davidson B., Nussbaum M., Lumbar extensor fatigue changes postural recovery strategy. Gait \& Posture. April 2006; 23(3):348-354.

[16] Schmidt, R.A., 1975. A schema theory of discrete motor skill learning. Psychol. Rev. 82, 225-260.

[17] Taylor, J.L., Butler, J.E., Gandevia, S.C., 2000. Changes in muscle afferents, motoneurons and motor drive during muscle fatigue. Eur. J.Appl. Physiol. 83, 106-115.

[18] Mezaour M, Yiou E, Le Bozec S. Effect of lower limb muscle fatigue on anticipatory postural adjustments associated with bilateral-forward reach in the unipedal dominant and non-dominant stance.Eur $\mathrm{J}$ Appl Physiol. 2010 Dec;110(6):1187-97.

[19] Giselle Delgado, Chelsea Coghlin, Katelyn Earle, Andrea Holek, and Kate O'Hare. Trunk Extensor Muscle Fatigue Does Not Affect Postural Control During Upright Static Stance in Young-Adults and Middle-Aged Adults. Western Undergraduate Research Journal: Health and Natural Sciences. Spring 2011; 2:17.

\section{Volume 6 Issue 7, July 2017 www.ijsr.net}




\section{International Journal of Science and Research (IJSR) \\ ISSN (Online): 2319-7064 \\ Index Copernicus Value (2015): 78.96 | Impact Factor (2015): 6.391}

[20] Bohannon R, Larkin P, Cook A, Gear J, Singer J. Decrease in timed balance test scores with aging. Physical Therapy. 1984; 64(7):1067-70.

[21] Duncan P., Weiner D. Functional reach: a new clinical measure of balance. J Gerontol. 1990; 45(6): M192-197.

[22]Borg G. Perceived exertion. Exercise Sports Science Revision. 1974; 2:131-153.

[23] MacDonalda D., Moseleyb L., Hodgesa P. The lumbar multifidus: Does the evidence support clinical beliefs? Manual Therapy. February 200611 (2006) 254-263

[24] Moseley GL, Hodges PW, Gandevia SC. Deep and superficial fibers of the lumbar multifidus muscle are differentially active during voluntary arm movements. Spine. 2002;27:E29-E36.

[25] Taimela S, Kankaanpää M, Luoto S. The effect of lumbar fatigue on the ability to sense a change in lumbar position. A controlled study. Spine (Phila Pa 1976). 1999 Jul 1;24(13):1322-7.

[26] Radebold A., Cholewicki J., Polzhofer G., Greene H. Impaired postural control of the lumbar spine is associated with delayed muscle response times in patients with chronic idiopathic low back pain. Spine.2001;26(7):724-730

[27] Allum J., Bloem B., Carpenter M. ,Hulliger M., Hadders-Algra M. Proprioceptive control of posture: a review of new concepts. Gait and Posture 8 (1998) 214242.

[28] Bloem B., Allum J., Carpenter MG, Honegger F (2000) Is lower leg proprioception essential for triggering human automatic postural responses? Experimental Brain Research 130, 3: 375-391.

[29] Riemann B., Myers J., Lephart S. Comparison of the ankle, knee, hip, and trunk corrective action shown during single-leg stance on firm, foam, and multiaxial surfaces. Arch Phys Med Rehabil.2003;84:90-5.

[30] Schieppati M, Nardone A, Schmid M. Neck muscle fatigue affects postural control in man. Neuroscience. 2003;121(2):277-85.

[31] GosselinG., RassoulianH., Brown I. Effects of neck extensor muscles fatigue on balance. Clinical Biomechanics. June 2004;19(5):473-479

[32] Liang Z., Clark R., Bryant A., June Quek J., Pua Y., Neck musculature fatigue affects specific frequency bands of postural dynamics during quiet standing. Gait \&posture.September 2013

[33] Vuillerme N., Nougier V., Teasdale N. Effects of lower limbs muscular fatigue on anticipatory postural adjustments during arm motions in humans. The journal of Sports Medicine and Physical fitness. 2002:42(3):289-294

[34] Mezaour M, Yiou E, Le Bozec S. Effect of lower limb muscle fatigue on anticipatory postural adjustments associated with bilateral-forward reach in the unipedal dominant and non-dominant stance. Eur J Appl Physiol. 2010 Dec;110(6):1187-97.

[35] Allison G.,Henry S. The influence of fatigue on trunk muscle responses to sudden arm movements, a pilot study. Clinical Biomechanics. 2002 July; 17(5):414-7.

[36] Strang A., Berg W. Fatigue-induced adaptive changes of anticipatory postural adjustments. Experimental Brain Research, March 2007;178(1):49-61

[37] Strang A., Berg W., Hieronymus M..Fatigue-induced early onset of anticipatory postural adjustments in non- fatigued muscles: support for a centrally mediated adaptation Experimental Brain Research, August 2009;197(3):245-254.

[38] Yiou E., Caderby T., Hussein T. Adaptability of anticipatory postural adjustments associated with voluntary movement. World J Orthop. 2012 June 18; 3(6):75-86.

[39] Granata K., Gottipati P. Fatigue influences the dynamic stability of the torso. Ergonomics. 2008; Aug;51(8):1258-71.

[40] Ritchie B, Woods J. Changes in muscle contractile properties and neural control during human muscular fatigue. Muscle Nerve. 1984;7:691-699.

[41] Søgaard K, Gandevia SC, Todd G, Petersen NT, Taylor JL. The effect of sustained low-intensity contractions on supraspinal fatigue in human elbow flexor muscles. $\mathrm{J}$ Physiol. 2006;573:511-523.

[42] Yaggie JA, McGregor SJ. Effects of isokinetic ankle fatigue on the maintenance of balance and postural limits. Arch Phys Med Rehabil. 2002 Feb;83(2):224-8.

[43] Nardone A, Tarantola J, Galante M, Schieppati M. Time course of stabilometric changes after a strenuous treadmill exercise. Arch Phys Med Rehabil. 1998;79: 920-924 